\title{
On a Grouping Method for Constructing Mixed Orthogonal Arrays
}

\author{
Chung-Yi Suen \\ Department of Mathematics, Cleveland State University, Cleveland, USA \\ Email: c.suen@csuohio.edu
}

Received January 20, 2012; revised February 19, 2012; accepted March 8, 2012

\section{ABSTRACT}

Mixed orthogonal arrays of strength two and size $s^{m n}$ are constructed by grouping points in the finite projective geometry $P G(m n-1, s) . P G(m n-1, s)$ can be partitioned into $\left[\left(s^{m n}-1\right) /\left(s^{n}-1\right)\right](n-1)$-flats such that each $(n-1)$ flat is associated with a point in $P G\left(m-1, s^{n}\right)$. An orthogonal array $L_{s^{n n}}\left(\left(s^{n}\right)^{\left(s^{m n}-1\right) /\left(s^{n}-1\right)}\right)$ can be constructed by using $\left(s^{m n}-1\right) /\left(s^{n}-1\right)$ points in $P G\left(m-1, s^{n}\right)$. A set of $\left(s^{t}-1\right) /(s-1)$ points in $P G\left(m-1, s^{n}\right)$ is called a $(t-1)$ flat over $G F(s)$ if it is isomorphic to $P G(t-1, s)$. If there exists a $(t-1)$-flat over $G F(s)$ in $P G\left(m-1, s^{n}\right)$, then we can replace the corresponding $\left[\left(s^{t}-1\right) /(s-1)\right] s^{n}$-level columns in $L_{s^{m n}}\left(\left(s^{n}\right)^{\left(s^{m n}-1\right) /\left(s^{n}-1\right)}\right)$ by $\left[\left(s^{n}-1\right) /(s-1)\right]$ $s^{t}$-level columns and obtain a mixed orthogonal array. Many new mixed orthogonal arrays can be obtained by this procedure. In this paper, we study methods for finding disjoint $(t-1)$-flats over $G F(s)$ in $P G\left(m-1, s^{n}\right)$ in order to construct more mixed orthogonal arrays of strength two. In particular, if $m$ and $n$ are relatively prime then we can construct an $L_{s^{m n}}\left(\left(s^{m}\right)^{\frac{s^{m n}-1}{s^{m}-1}}-\frac{i\left(s^{n}-1\right)}{s-1}\left(s^{n}\right)^{\frac{i\left(s^{m}-1\right)}{s-1}}\right)$ for any $0 \leq i \leq \frac{\left(s^{m n}-1\right)(s-1)}{\left(s^{m}-1\right)\left(s^{n}-1\right)}$. New orthogonal arrays of sizes 256, 512, and 1024 are obtained by using $P G(7,2), P G(8,2)$ and $P G(9,2)$ respectively.

Keywords: Finite Field; Finite Projective Geometry; $(t-1)$-Flat over $G F(\mathrm{~s})$ in $P G\left(m-1, s^{n}\right)$; Geometric Orthogonal Array; Matrix Representation; Minimal Polynomial; Orthogonal Main-Effect Plan; Primitive Element; Tight

\section{Introduction}

Orthogonal arrays of strength two are used as orthogonal main-effect plans in fractional factorial experiments. In an orthogonal main-effect plan, the main effects of each factor can be optimally estimated assuming the interactions of all factors are negligible.

Let $L_{N}\left(s_{1} \cdots s_{k}\right)$ denote an orthogonal arrays of strength two with $N$ rows, $k$ columns, and $s_{i}$ levels in the $i$ th column for $i=1, \cdots, k$. In every $N \times 2$ subarray of $L_{N}\left(s_{1} \cdots s_{k}\right)$, all possible combinations of levels occur equally often as rows. It is known that $N-1 \geq \sum\left(s_{i}-1\right)$ in an $L_{N}\left(s_{1} \cdots s_{k}\right)$ and the orthogonal array is called tight if the equality holds. Orthogonal array $L_{N}\left(s_{1} \cdots s_{k}\right)$ is called symmetric if $s_{1}=\cdots=s_{k}$, otherwise it is called asymmetric or mixed. Symmetric orthogonal arrays have been constructed in [1-3]. Mixed orthogonal arrays were introduced in [4], and they have drawn the attentions of many researchers in recent years. Methods for constructing mixed orthogonal arrays of strength two have been developed in [5-9], and many other authors. These methods use Hadamard matrices, difference schemes, generalized Kronecker sums, finite projective geometries, and orthogonal projection matrices. We refer to [10] for more constructions and applications of orthogonal arrays.

The method of grouping was used in [11] to replace three two-level columns in symmetric orthogonal arrays by one four-level column for constructing mixed orthogonal arrays having two-level and four-level columns. A systematic method [12] was developed for identifying 
disjoint sets of three two-level columns for constructing $L_{N}\left(2^{m} 4^{n}\right)$. The method was generalized in [6] for constructing $L_{s^{k}}\left(s^{m}\left(s^{r_{1}}\right)^{n_{1}} \cdots\left(s^{r_{t}}\right)^{n_{t}}\right)$, where $s$ is a prime power. Mixed orthogonal arrays of strength $t$ were constructed by using mixed spreads of strength $t$ in finite geometries in [13]. This method was also independently discovered in [14] for constructing mixed orthogonal arrays of strength three and four. Orthogonal arrays constructed by this method are called geometric. Geometric orthogonal arrays $L_{64}\left(8^{6} 4^{7}\right), L_{64}\left(8^{3} 4^{14}\right), L_{64}\left(8^{4} 4^{10} 2^{5}\right)$ and $L_{64}\left(8^{1} 4^{17} 2^{5}\right)$ were constructed in [13]. However, the method is restricted to constructing mixed orthogonal arrays with the number of levels in each column a power of 2 . In this paper, we shall use finite projective geometries to develop a general procedure for constructing more mixed orthogonal arrays. Moreover, the procedure allows us to construct mixed orthogonal arrays with the number of levels in each column a power of any given prime number. We start with a symmetric orthogonal array $L_{s^{m n}}\left(\left(s^{n}\right)^{\left(s^{m n}-1\right) /\left(s^{n}-1\right)}\right)$, and then construct mixed orthogonal arrays by replacing a group of columns with another group of columns. Our grouping method uses properties of finite projective geometries, which is different from the grouping method in [6]. Hence we are able to obtain some new series of mixed orthogonal arrays.

\section{Geometric Orthogonal Arrays}

For $r \geq 1$ and $s$ a prime power, let $P G(r-1, s)$ denote the $(r-1)$-dimensional finite projective geometry over the Galois field $G F(s)$. A point in $P G(r-1, s)$ is denoted by an $r$-tuple $\left(x_{1}, \cdots, x_{r}\right)$, where $x_{i}$ 's are elements of $G F(s)$ and at least one $x_{i}$ is not 0 . Two $r$-tuples represent the same point in $P G(r-1, s)$ if one is a multiple of the other. Hence there are $\left(s^{r}-1\right) /(s-1)$ points in $P G(r-1, s)$. A $(t-1)$-flat in $P G(r-1, s)$ is a set of $\left(s^{t}-1\right) /(s-1)$ points which are linear combinations of $t$ independent points. A spread $\mathcal{F}$ of $(t-1)$-flats of $P G(r-1, s)$ is a set of $(t-1)$-flats which partition $P G(r-1, s)$. It is known [15] that there exists a spread $\mathcal{F}$ of $(t-1)$-flats of $P G(r-1, s)$ if and only if $t$ divides $r$.

We call a set of flats $\mathcal{F}=\left\{F_{1}, \cdots, F_{k}\right\}$ a mixed spread of $P G(r-1, s)$ if it partitions $P G(r-1, s)$ and at least two flats in $\mathcal{F}$ have different dimensions. Mixed spreads are useful for constructing mixed orthogonal arrays of strength two. Specifically, we give the following theorem for constructing an orthogonal array from a (mixed) spread. The theorem is the special case of strength two of Theorem 2.1 [14] in finite projective geometry's language.
Theorem 1. Let $\mathcal{F}=\left\{F_{1}, \cdots, F_{k}\right\}$ be a (mixed) spread of $P G(r-1, s)$, where $F_{i}$ is a $\left(t_{i}-1\right)$-flat for

$i=1, \cdots, k$. Then we can construct an orthogonal array $L_{s^{r}}\left(\left(s^{t_{1}}\right) \cdots\left(s^{t_{k}}\right)\right)$.

We now describe the procedure to construct the orthogonal array in Theorem 1 . For $i=1, \cdots, k$, let $G_{i}$ be an $r \times t_{i}$ matrix such that the $t_{i}$ columns are any choice of $t_{i}$ independent points of the $\left(t_{i}-1\right)$-flat $F_{i}$. Let $G$ be the $r \times \sum t_{i}$ matrix $\left[G_{1}, \cdots, G_{k}\right]$. The $L_{s^{r}}\left(\left(s^{t_{1}}\right) \cdots\left(s^{t_{k}}\right)\right)$ consists of $s^{r}$ rows which are the elements of the row space of $G$, where the $t_{i} s$-level columns of $G_{i}$ is replaced by an $s^{t_{i}}$-level column for each $i=1, \cdots, k$. We call orthogonal arrays geometric if they can be obtained by Theorem 1 . Geometric orthogonal arrays have been constructed in [1, $9,13,16]$. Examples of geometric orthogonal arrays are:
1) $L_{s^{r}}\left(s^{\left(s^{r}-1\right) /(s-1)}\right)$;
2) $L_{s^{r}}\left(\left(s^{t}\right)^{\left(s^{r}-1\right) /\left(s^{t}-1\right)}\right)$ if $t$ divides $r$;
3) $L_{s^{r}}\left(\left(s^{r-t}\right)^{1}\left(s^{t}\right)^{s^{r-t}}\right)$ if $r \geq 2 t$; and
4) $L_{s^{r}}\left(\left(s^{t}\right)^{k} s^{l}\right)$, where,

$$
\begin{gathered}
k=s^{j}\left(s^{i t}-1\right) /\left(s^{t}-1\right)-s^{j}+1, \\
l=s^{t}\left(s^{j}-1\right) /(s-1), \quad r=i t+j, \quad 0 \leq j<t .
\end{gathered}
$$

\section{Main Results}

It is proved in Lemma 12 [13] that if $V_{1}, V_{2}, V_{3}$ are three disjoint $(n-1)$-flats of $P G(2 n-1,2)$ then their union can be regrouped into $2^{n}-1$ disjoint 1 -flats. Hence three $2^{n}$-level columns in an $L_{2^{2 n}}\left(\left(2^{n}\right)^{2^{n}+1}\right)$ can be replaced by $\left(2^{n}-1\right) 4$-level columns. By applying this result to a spread of 2-flats of $P G(5,2), L_{64}\left(8^{6} 4^{7}\right)$ and $L_{64}\left(8^{3} 4^{14}\right)$ were constructed. Generalizing the idea, we would like to find a sufficient condition that a set of $\left[\left(s^{t}-1\right) /(s-1)\right]$ $(n-1)$-flats in $P G(m n-1, s)$ can be regrouped into a set of $\left[\left(s^{n}-1\right) /(s-1)\right](t-1)$-flats.

Since there exists a spread of $(n-1)$-flats of $P G(m n-1, s)$, we can, by Theorem 1 , construct an $L_{s^{m n}}\left(\left(s^{n}\right)^{\left(s^{m n}-1\right) /\left(s^{n}-1\right)}\right)$. If there exist $\left[\left(s^{t}-1\right) /(s-1)\right]$ $(n-1)$-flats in the spread such that their union can be regrouped into $\left[\left(s^{n}-1\right) /(s-1)\right](t-1)$-flats, then we can replace the corresponding $\left[\left(s^{t}-1\right) /(s-1)\right] s^{n}$-level 
columns in the $L_{s^{m n}}\left(\left(s^{n}\right)^{\left(s^{m n}-1\right) /\left(s^{n}-1\right)}\right)$ by $\left[\left(s^{n}-1\right) /(s-1)\right] s^{t}$-level columns and obtain an $L_{s^{m n}}\left(\left(s^{n}\right)^{\frac{s^{m n}-1}{s^{n}-1}-\frac{s^{t}-1}{s-1}}\left(s^{t}\right)^{\frac{s^{n}-1}{s-1}}\right)$. By repeating this process, many orthogonal arrays can be obtained.

First we would like to establish a one-to-one correspondence between the $\left(s^{m n}-1\right) /\left(s^{n}-1\right)$ disjoint $(n-1)$ flats in $P G(m n-1, s)$ and the $\left(s^{m n}-1\right) /\left(s^{n}-1\right)$ points in $P G\left(m n-1, s^{n}\right)$. Let $\omega$ be a primitive element of $G F\left(s^{n}\right)$ and let the minimum polynomial of $G F\left(s^{n}\right)$ be $\omega^{n}+\alpha_{n-1} \omega^{n-1}+\cdots+\alpha_{1} \omega+\alpha_{0}$, where $\alpha_{0}, \cdots, \alpha_{n-1}$ are elements of $G F(s)$. The companion matrix of the minimum polynomial is an $n \times n$ matrix

$$
\boldsymbol{W}=\left[\begin{array}{ccccc}
0 & 1 & 0 & \cdots & 0 \\
0 & 0 & 1 & \cdots & 0 \\
\vdots & \vdots & \vdots & \ddots & \vdots \\
0 & 0 & 0 & \cdots & 1 \\
-\alpha_{0} & -\alpha_{1} & -\alpha_{2} & \cdots & -\alpha_{n-1}
\end{array}\right] .
$$

If $\omega$ is a primitive element of $G F\left(s^{n}\right)$, then $0,1, \omega, \cdots, \omega^{s^{n}-2}$ are the $s^{n}$ elements of $G F\left(s^{n}\right)$. The elements of $G F\left(s^{n}\right)$ can be represented by $n \times n$ matrices with entries from $G F(s)$. The element $\omega^{i}$ is represented by $W^{i}$, and the elements 0 and 1 are represented by the zero matrix and the identity matrix respectively. Denote the matrix representation of an element $x$ in $G F\left(s^{n}\right)$ by $W(x)$. Let each point $\left(x_{1}, \cdots, x_{m}\right)$ in $P G\left(m-1, s^{n}\right)$ correspond to the $(n-1)$-flat in $P G(m n-1, s)$ which consists of points that are linear combinations of row vectors of the $n \times m n$ matrix $\left[W\left(x_{1}\right), \cdots, W\left(x_{m}\right)\right]$ over $G F(s)$. It can be shown that the $\left[\left(s^{m n}-1\right) /\left(s^{n}-1\right)\right](n-1)$-flats corresponding to the $\left[\left(s^{m n}-1\right) /\left(s^{n}-1\right)\right]$ points of $P G\left(m-1, s^{n}\right)$ partition $P G(m n-1, s)$. This establishes a one-to-one correspondence between the $\left(s^{m n}-1\right) /\left(s^{n}-1\right)$ disjoint $(n-1)$-flats in $P G(m n-1, s)$ and the $\left(s^{m n}-1\right) /\left(s^{n}-1\right)$ points in $P G\left(m-1, s^{n}\right)$.

Definition 1. A set of $\left(s^{t}-1\right) /(s-1)$ points in $P G\left(m-1, s^{n}\right)$ is said to be a $(t-1)$-flat over $G F(s)$ if it is possible to find coordinates for this set of $\left(s^{t}-1\right) /(s-1)$ points such that it is isomorphic to $P G(t-1, s)$ over $G F(s)$.
Note that whether a set of $\left(s^{t}-1\right) /(s-1)$ points in $P G\left(m-1, s^{n}\right)$ is isomorphic to $P G(t-1, s)$ over $G F(s)$ depends not only on the choice of the points but also on the choice of the coordinates for these points. For example, the set $S_{1}=\left\{(1, \omega),\left(\omega, \omega^{3}\right),\left(\omega^{3}, 1\right)\right\}$ in Example 1 (given after Theorem 2) is an 1-flat over $G F(2)$ in $P G(1,8)$ since it is isomorphic to $P G(1,2)$ over $G F(2)$. But if we choose different coordinates for $S_{1}=\left\{(1, \omega),\left(1, \omega^{2}\right),\left(1, \omega^{4}\right)\right\}$, then it is not isomorphic to $P G(1,2)$ over $G F(2)$. Hence it is important to specify the correct coordinates when a $(t-1)$-flat over $G F(s)$ in $P G\left(m-1, s^{n}\right)$ is mentioned. Also we note that it is possible to have $t>m$ for a $(t-1)$-flat over $G F(s)$ in $P G\left(m-1, s^{n}\right)$. For example, $S_{1}$ and $S_{2}$ in Example 2 (given after Theorem 2) are 2-flats over $G F(2)$ in $P G(1,16)$.

We now give a sufficient condition that a set of $\left(s^{t}-1\right) /(s-1)$ disjoint $(n-1)$-flats in $P G(m n-1, s)$ can be regrouped into a set of $\left(s^{n}-1\right) /(s-1)$ disjoint $(t-1)$ - flats.

Theorem 2. A set of $\left(s^{t}-1\right) /(s-1)$ disjoint $(n-1)$ flats in $P G(m n-1, s)$ can be regrouped into a set of $\left(s^{n}-1\right) /(s-1)$ disjoints $(t-1)$-flats, if the set of $\left(s^{t}-1\right) /(s-1)$ corresponding points in $P G\left(m-1, s^{n}\right)$ is a $(t-1)$-flat over $G F(s)$.

Proof. Let the coordinates of the $\left(s^{t}-1\right) /(s-1)$ corresponding points of the $(t-1)$-flat over $G F(s)$ in $P G\left(m-1, s^{n}\right)$ be $\left(x_{1 j}, \cdots, x_{m j}\right)$ for $j=1, \cdots,\left(s^{t}-1\right) /(s-1)$. Also let $L$ be an $\left[\left(s^{n}-1\right) /(s-1)\right] \times n$ matrix such that the rows are the points of $P G(n-1, s)$. Then the $(n-1)$-flat in $P G(m n-1, s)$ corresponding to the point $\left(x_{1 j}, \cdots, x_{m j}\right)$ consists of points which are the rows of the $\left[\left(s^{n}-1\right) /(s-1)\right] \times m n$ matrix $M_{j}=L\left[W\left(x_{1 j}\right), \cdots, W\left(x_{m j}\right)\right]$, where $W(x)$ is the $n \times n$ matrix representation of $x$. We can verify that for each $i=1, \cdots,\left(s^{n}-1\right) /(s-1)$, the set of $\left(s^{t}-1\right) /(s-1)$ points which consists of the $i$ th rows of $M_{1}, \cdots, M_{\left(s^{t}-1\right) /(s-1)}$ is a
$(t-1)$-flat in $P G(m n-1, s)$.

Note that in general there are more ways of regrouping a set of $\left(s^{t}-1\right) /(s-1)$ disjoint $(n-1)$-flats in 
$P G(m n-1, s)$ into disjoint flats if the $\left(s^{t}-1\right) /(s-1)$ corresponding points in $P G\left(m-1, s^{n}\right)$ is a $(t-1)$-flat over $G F(s)$. Let $P_{i j}$ be the point in $P G(m n-1, s)$ with the $i$ th row of $M_{j}$ as its coordinates. The $\left[\left(s^{n}-1\right) /(s-1)\right] \times\left[\left(s^{t}-1\right) /(s-1)\right]$ array of points $\mathbf{P}=\left[P_{i j}\right]$ has the following properties: flat).

1) Each row (column) of $\mathbf{P}$ is a $(t-1)$-flat $((n-1)$ 2) If $\left(s^{u}-1\right) /(s-1)$ points in a given row (column) form a $(u-1)$-flat, then the $\left(s^{u}-1\right) /(s-1)$ points at the same positions in any other row (column) also form a $(u-1)$-flat.

For example, if there exists a 2-flat over $G F(2)$ in $P G(1,16)$, then each of the 7 points in the 2-flat over $G F(2)$ corresponds to 15 points in $P G(7,2)$. The 105 points in $P G(7,2)$ corresponding to the 2-flat over $G F(2)$ in $P G(1,16)$ can be arranged into a $15 \times 7$ array such that each row is a 2-flat and each column is a 3-flat. Since a 3 -flat can be partitioned into five 1-flats, the $15 \times 7$ array of points can be partitioned into five $3 \times 7$ subarrays such that each column is a 1-flat and each row is a 2-flat. Also, consider a $15 \times 3$ subarray of the $15 \times 7$ array such that each row is a 1 -flat. We can select a $7 \times 3$ subarray such that each column is a 2-flat. Each of the remaining eight rows is a 1-flat. Hence the $15 \times 3$ subarray can be partitioned into three 2-flat and eight 1-flats. Therefore, these 105 points can be grouped into: 1$)(7-i) 3$-flats and $5 i$ 1-flats for $i=0, \cdots, 7 ; 2)(15-3 i)$ 2-flats and $7 i$ 1-flats for $i=0, \cdots, 5$; or 3 ) four 3 -flats, three 2 -flats, and eight 1-flats.

Example 1. Let $0,1, \omega, \cdots, \omega^{6}$ be the 8 elements of $G F(8)$ with $\omega^{3}=\omega+1$. Consider $P G(1,8)$ with nine points $(0,1),(1,0),(1,1),(1, \omega),\left(1, \omega^{2}\right),\left(1, \omega^{3}\right),\left(1, \omega^{4}\right)$, $\left(1, \omega^{5}\right)$ and $\left(1, \omega^{6}\right)$. Each point of $P G(1,8)$ corresponds to a 2-flat in $P G(5,2)$, and the nine 2-flats partition $P G(5$, 2 ). We can construct an $L_{64}\left(8^{9}\right)$ by Theorem 1 . Let

$$
\begin{aligned}
& S_{1}=\left\{(1, \omega),\left(\omega, \omega^{3}\right),\left(\omega^{3}, 1\right)\right\}, \\
& S_{2}=\left\{\left(1, \omega^{3}\right),\left(\omega^{3}, \omega\right),(\omega, 1)\right\}, \text { and } \\
& S_{3}=\{(0,1),(1,0),(1,1)\} .
\end{aligned}
$$

We can verify that $S_{1}, S_{2}$, and $S_{3}$ are disjoint 1 -flats over $G F(2)$ in $P G(1,8)$. The $3 \times 3$ matrix representation $W$ of $\omega$ and the $7 \times 3$ matrix $L$ given in the proof of Theorem 2 are

$$
\boldsymbol{W}=\left[\begin{array}{lll}
0 & 1 & 0 \\
0 & 0 & 1 \\
1 & 1 & 0
\end{array}\right] ; \quad \boldsymbol{L}=\left[\begin{array}{lllllll}
1 & 0 & 0 & 1 & 0 & 1 & 1 \\
0 & 1 & 0 & 1 & 1 & 1 & 0 \\
0 & 0 & 1 & 0 & 0 & 1 & 1
\end{array}\right]^{\mathrm{T}} \text {. }
$$

The three points of $S_{1}$ correspond to the three 2-flats in $P G(5,2)$ which are rows of the following three matrices $\boldsymbol{M}_{1}, \boldsymbol{M}_{2}$, and $\boldsymbol{M}_{3}$ respectively.

$$
\begin{aligned}
& \boldsymbol{M}_{1}=\boldsymbol{L}[W(1), W(\omega)]=\left[\begin{array}{llllll}
1 & 0 & 0 & 0 & 1 & 0 \\
0 & 1 & 0 & 0 & 0 & 1 \\
0 & 0 & 1 & 1 & 1 & 0 \\
1 & 1 & 0 & 0 & 1 & 1 \\
0 & 1 & 1 & 1 & 1 & 1 \\
1 & 1 & 1 & 1 & 0 & 1 \\
1 & 0 & 1 & 1 & 0 & 0
\end{array}\right], \\
& \boldsymbol{M}_{2}=\boldsymbol{L}\left[W(\omega), W\left(\omega^{3}\right)\right]=\left[\begin{array}{llllll}
0 & 1 & 0 & 1 & 1 & 0 \\
0 & 0 & 1 & 0 & 1 & 1 \\
1 & 1 & 0 & 1 & 1 & 1 \\
0 & 1 & 1 & 1 & 0 & 1 \\
1 & 1 & 1 & 1 & 0 & 0 \\
1 & 0 & 1 & 0 & 1 & 0 \\
1 & 0 & 0 & 0 & 0 & 1
\end{array}\right] \text {, } \\
& \boldsymbol{M}_{3}=\boldsymbol{L}\left[W\left(\omega^{3}\right), W(1)\right]=\left[\begin{array}{llllll}
1 & 1 & 0 & 1 & 0 & 0 \\
0 & 1 & 1 & 0 & 1 & 0 \\
1 & 1 & 1 & 0 & 0 & 1 \\
1 & 0 & 1 & 1 & 1 & 0 \\
1 & 0 & 0 & 0 & 1 & 1 \\
0 & 1 & 0 & 1 & 1 & 1 \\
0 & 0 & 1 & 1 & 0 & 1
\end{array}\right] .
\end{aligned}
$$

We observe that for each $i=1, \cdots, 7$, the $i$ th rows of $\boldsymbol{M}_{1}, \boldsymbol{M}_{2}$, and $\boldsymbol{M}_{3}$ are three points of a 1-flat in $P G(5,2)$. Hence we can replace the three 8-level columns corresponding to $S_{1}$ in $L_{64}\left(8^{9}\right)$ by seven 4 -level columns to obtain an $L\left(8^{6} 4^{7}\right)$. Continuing this procedure, we can replace the three 8-level columns corresponding to $S_{2}$ in $L_{64}\left(8^{6} 4^{7}\right)$ by seven 4 -level columns to obtain an $L_{64}\left(8^{3} 4^{14}\right)$. $\square$

Note that $L_{64}\left(8^{6} 4^{7}\right)$ and $L_{64}\left(8^{3} 4^{14}\right)$ were also construct in [13] using a different method. However, Theorem 2 is more versatile as shown in following example.

Example 2. Let $0,1, \omega, \cdots, \omega^{14}$ be the 16 elements of $G F(16)$ with $\omega^{4}=\omega+1$. Consider $P G(1,16)$ with 17 points $(0,1),(1,0),(1,1),(1, \omega), \cdots,\left(1, \omega^{14}\right)$. Each point of $P G(1,16)$ corresponds to a 3-flat in $P G(7,2)$, and the seventeen 3 -flats partition $P G(7,2)$. We can construct an $L_{256}\left(16^{17}\right)$ by Theorem 1 . Let

$$
\begin{aligned}
& S_{1}=\left\{\left(1, \omega^{7}\right),\left(\omega, \omega^{9}\right),\left(\omega^{2}, \omega^{12}\right),\left(\omega^{4}, 1\right),\left(\omega^{5}, \omega^{8}\right),\left(\omega^{8}, \omega^{2}\right),\left(\omega^{10}, \omega^{11}\right)\right\}, \\
& S_{2}=\left\{\left(1, \omega^{12}\right),\left(\omega, \omega^{3}\right),\left(\omega^{2}, \omega\right),\left(\omega^{4}, \omega^{10}\right),\left(\omega^{5}, \omega^{9}\right),\left(\omega^{8}, \omega^{13}\right),\left(\omega^{10}, \omega^{8}\right)\right\},
\end{aligned}
$$




$$
\begin{aligned}
& T_{1}=\{(0,1),(1,0),(1,1)\}, T_{2}=\left\{(1, \omega),\left(\omega, \omega^{4}\right),\left(\omega^{4}, 1\right)\right\}, T_{3}=\left\{\left(1, \omega^{2}\right),\left(\omega^{2}, \omega^{8}\right),\left(\omega^{8}, 1\right)\right\}, \\
& T_{4}=\left\{\left(1, \omega^{4}\right),\left(\omega^{4}, \omega\right),(\omega, 1)\right\}, \text { and } T_{5}=\left\{\left(1, \omega^{8}\right),\left(\omega^{8}, \omega^{2}\right),\left(\omega^{2}, 1\right)\right\} .
\end{aligned}
$$

We can verify that $S_{1}$ and $S_{2}$ are disjoint 2-flats and $T_{1}, \cdots, T_{5}$ are disjoint 1-flats over $G F(2)$ in $P G(1,16)$. Moreover, $S_{1}, S_{2}$, and $T_{1}$ partition $P G(1,16)$. By the discussion following Theorem 2 , we can replace the subarray $L_{256}\left(16^{7}\right)$ corresponding to $S_{1}$ or $S_{2}$ in $L_{256}\left(16^{17}\right)$ by $L_{256}\left(16^{4} 8^{3} 4^{8}\right)$ or $L_{256}\left(8^{15-3 i} 4^{7 i}\right), 0 \leq i \leq 5$. Similarly, we can replace the subarray $L_{256}\left(16^{3}\right)$ corresponding to $T_{1}, \cdots, T_{5}$ in $L_{256}\left(16^{17}\right)$ by $L_{256}\left(8^{3} 4^{8}\right)$. Many mixed orthogonal arrays such as $L_{256}\left(16^{10} 8^{15}\right), L_{256}\left(16^{3} 8^{30}\right), L_{256}\left(16^{10} 8^{12} 4^{7}\right)$, $L_{256}\left(16^{10} 8^{9} 4^{14}\right), L_{256}\left(16^{14} 8^{3} 4^{8}\right), L_{256}\left(16^{7} 8^{18} 4^{8}\right)$, $L_{256}\left(16^{11} 8^{6} 4^{16}\right), \cdots$, can be obtained by this procedure. $\square$

\section{Construction of More Orthogonal Arrays}

In this section, methods for finding disjoint flats over $G F(s)$ in $P G\left(m-1, s^{n}\right)$ are developed to construct more orthogonal arrays. Let $\alpha$ be a primitive element of $G F\left(s^{m}\right)$, and let the $m \times m$ matrix representation of $\alpha$ in $G F(s)$ be $W$. Since $\alpha^{\left(s^{m}-1\right) /(s-1)}$ is an element of $G F(s)$ and $W^{\left(s^{m}-1\right) /(s-1)}$ is the matrix representation of $\alpha^{\left(s^{m}-1\right) /(s-1)}$, we have $W^{\left(s^{m}-1\right) /(s-1)}=\alpha^{\left(s^{m}-1\right) /(s-1)} I_{m}$, where $I_{m}$ is the $m \times m$ identity matrix. Then for any fixed point $x=\left(x_{1}, \cdots, x_{m}\right)$ in $P G\left(m-1, s^{n}\right)$, the set

$S_{x}=\left\{x W^{i}: i \geq 0\right\}$ contains at $\operatorname{most}\left(s^{m}-1\right) /(s-1)$ points in $P G\left(m-1, s^{n}\right)$ since $x W^{\left(s^{m}-1\right) /(s-1)}$

$\left(=x \alpha^{\left(s^{m}-1\right) /(s-1)} I_{m}=\alpha^{\left(s^{m}-1\right) /(s-1)} x\right)$ and $x$ represent the same point. Moreover, if $\beta$ and $\gamma$ are any elements of $G F(s)$ and $x W^{i}$ and $x W^{j}$ are elements of $S_{x}$, then $\beta x W^{i}+\gamma x W^{j}=x\left(\beta W^{i}+\gamma W^{j}\right)=x W^{l}$ for some $l$, since $\beta W^{i}+\gamma W^{j}$ is the matrix representation of the element $\beta \alpha^{i}$ $+\gamma \alpha^{j}$ of $G F\left(s^{m}\right) . S_{x}$ has the structure of a flat over $G F(s)$ in $P G\left(m-1, s^{n}\right)$ since linear combinations of points in $S_{x}$ are also points in $S_{x}$. In fact, $S_{x}$ is a $(t-1)$-flat over $G F(s)$ in $P G\left(m-1, s^{n}\right)$ if and only if the number of points in $S_{x}$ is $\left(s^{t}-1\right) /(s-1)$ for some integer $t$. Now if $x$ and $y$ are two points in $P G\left(m-1, s^{n}\right)$ and

$S_{x} \cap S_{y} \neq \phi$, then there exist $i$ and $j$ such that $x W^{i}=y W^{j}$. We have $y=x W^{i-j} \in S_{x}$, hence $S_{x}=S_{y}$.

Theorem 3. Let $x$ be a point in $P G\left(m-1, s^{n}\right)$, and let $S_{x}=\left\{x W^{i}: i \geq 0\right\}$. Then $S_{x}$ is a $(t-1)$-flat over $G F(s)$ in $P G\left(m-1, s^{n}\right)$ if and only if the number of points in $S_{x}$ is $\left(s^{t}-1\right) /(s-1)$ for some integer $t$. Moreover, for any two points $x$ and $y$ in $P G\left(m-1, s^{n}\right)$ either $S_{x}=S_{y}$ or $S_{x} \cap S_{y}=\phi$. Hence $P G\left(m-1, s^{n}\right)$ can be partitioned into disjoint sets of $S_{x}$ 's.

Example 3. We illustrate how we obtain the three disjoint 1-flats over $G F(2)$ in $P G(1,8)$ in Example 1. Let $\omega$ be a primitive element of $G F(8)$ with $\omega^{3}=\omega+1$, and let $\alpha$ be a primitive element of $G F(4)$ with $\alpha^{2}=\alpha+1$ and matrix representation

$$
W=\left[\begin{array}{ll}
0 & 1 \\
1 & 1
\end{array}\right] .
$$

Then

$$
\begin{aligned}
& S_{(0,1)}=\left\{(0,1),(0,1) W,(0,1) W^{2}\right\}=\{(0,1),(1,1),(1,0)\}, \\
& S_{(1, \omega)}=\left\{(1, \omega),(1, \omega) W,(1, \omega) W^{2}\right\}=\left\{(1, \omega),\left(\omega, \omega^{3}\right),\left(\omega^{3}, 1\right)\right\}, \text { and } \\
& S_{\left(1, \omega^{3}\right)}=\left\{\left(1, \omega^{3}\right),\left(1, \omega^{3}\right) W,\left(1, \omega^{3}\right) W^{2}\right\}=\left\{\left(1, \omega^{3}\right),\left(\omega^{3}, \omega\right),(\omega, 1)\right\}
\end{aligned}
$$

are three disjoint 1-flats over $G F(2)$ in $P G(1,8)$. $\square$

Example 4. Let $\omega$ be a primitive element of $G F(16)$ with $\omega^{4}=\omega+1$, and let $\alpha$ be a primitive element of $G F(4)$ with $\alpha^{2}=\alpha+1$ and matrix representation $W$ given in Example 3 . The 17 points of $P G(1,16)$ can be partitioned into the following flats over $G F(2)$ :

$$
\begin{aligned}
& S_{(0,1)}=\{(0,1),(1,1),(1,0)\}, \\
& S_{(1, \omega)}=\left\{(1, \omega),\left(\omega, \omega^{4}\right),\left(\omega^{4}, 1\right)\right\}, \\
& S_{\left(1, \omega^{2}\right)}=\left\{\left(1, \omega^{2}\right),\left(\omega^{2}, \omega^{8}\right),\left(\omega^{8}, 1\right)\right\},
\end{aligned}
$$

$$
\begin{aligned}
& S_{\left(1, \omega^{4}\right)}=\left\{\left(1, \omega^{4}\right),\left(\omega^{4}, \omega\right),(\omega, 1)\right\}, \\
& S_{\left(1, \omega^{8}\right)}=\left\{\left(1, \omega^{8}\right),\left(\omega^{8}, \omega^{2}\right),\left(\omega^{2}, 1\right)\right\}, \\
& S_{\left(1, \omega^{5}\right)}=\left\{\left(1, \omega^{5}\right)\right\}, \text { and } S_{\left(1, \omega^{10}\right)}=\left\{\left(1, \omega^{10}\right)\right\} .
\end{aligned}
$$

The five disjoint 1-flats over $G F(2)$ are $T_{1}, \cdots, T_{5}$ in Example 2. $\square$

Theorem 4. If $s$ is a prime power and $m$ and $n$ are relatively prime, then we can construct mixed orthogonal arrays 


$$
L_{s^{m n}}\left(\left(s^{n}\right)^{\left(s^{m n}-1\right) /\left(s^{n}-1\right)-i\left(s^{m}-1\right) /(s-1)}\left(s^{m}\right)^{i\left(s^{n}-1\right) /(s-1)}\right)
$$

for $i=0, \cdots,\left[\left(s^{m n}-1\right)(s-1)\right] /\left[\left(s^{m}-1\right)\left(s^{n}-1\right)\right]$.

Proof. We can construct an $L_{s^{m n}}\left(\left(s^{n}\right)^{\left(s^{m n}-1\right) /\left(s^{n}-1\right)}\right)$ from $P G\left(m-1, s^{n}\right)$. From the proof of Theorem 4.3.6 [15], if $m$ and $n$ are relatively prime then $S_{x}$ is an $(m-1)$-flat over $G F(s)$ in $P G\left(m-1, s^{n}\right)$ for every point $x$ in $P G\left(m-1, s^{n}\right)$. Hence $P G\left(m-1, s^{n}\right)$ can be partitioned into $\left[\left(s^{m n}-1\right)(s-1)\right] /\left[\left(s^{m}-1\right)\left(s^{n}-1\right)\right]$ $(m-1)$-flats over $G F(s)$. Each $S_{x}$ represents $\left[\left(s^{m}-1\right) /(s-1)\right] s^{n}$-level columns in $L_{s^{m n}}\left(\left(s^{n}\right)^{\left(s^{m n}-1\right) /\left(s^{n}-1\right)}\right)$, and by Theorem 2 it can be replaced by $\left(s^{n}-1\right) /(s-1) s^{m}$-level columns. $\square$

The following result which follows from Theorem 4 is a generalization of Theorem 4 .

Corollary 1. If $\mathrm{s}$ is a prime power and $d$ is the greatest common divisor of integers $m$ and $n$, then we can construct mixed orthogonal arrays

$$
L_{s^{m n / d}}\left(\left(s^{n}\right)^{\left(s^{m n}-1\right) /\left(s^{n}-1\right)-i\left(s^{m}-1\right) /\left(s^{d}-1\right)}\left(s^{m}\right)^{i\left(s^{n}-1\right) /\left(s^{d}-1\right)}\right)
$$

for $i=0, \cdots,\left[\left(s^{m n / d}-1\right)\left(s^{d}-1\right)\right] /\left[\left(s^{m}-1\right)\left(s^{n}-1\right)\right]$.

Proof. If $d$ is the greatest common divisor of $m$ and $n$, then $m / d$ and $n / d$ are relatively prime. By substituting $m, n$, and $s$ with $m / d, n / d$, and $s^{d}$ respectively in Theorem 4, we obtain the mixed orthogonal arrays. $\square$

By using Theorem 4 and Corollary 1, we obtain the following new series of tight orthogonal arrays for any prime power $s$.

$$
\begin{gathered}
\text { 1) } L_{s^{6}}\left(\left(s^{3}\right)^{\frac{s^{6}-1}{s^{3}-1}-\frac{i\left(s^{2}-1\right)}{s-1}}\left(s^{2}\right)^{\frac{i\left(s^{3}-1\right)}{s-1}}\right), \\
0 \leq i \leq s^{2}-s+1 ; \\
\text { 2) } L_{s^{10}}\left(\left(s^{5}\right)^{\frac{s^{10}-1}{s^{5}-1}-\frac{i\left(s^{2}-1\right)}{s-1}}\left(s^{2}\right)^{\frac{i\left(s^{5}-1\right)}{s-1}}\right), \\
0 \leq i \leq\left(s^{5}+1\right) /(s+1) ; \\
\text { 3) } L_{s^{12}}\left(\left(s^{4}\right)^{\frac{s^{12}-1}{s^{4}-1}-\frac{i\left(s^{3}-1\right)}{s-1}}\left(s^{3}\right)^{\frac{i\left(s^{4}-1\right)}{s-1}}\right), \\
0 \leq i \leq\left(s^{4}-s^{2}+1\right)\left(s^{2}-s+1\right) ;
\end{gathered}
$$

4) $L_{s^{12}}\left(\left(s^{6}\right)^{\frac{s^{12}-1}{s^{6}-1}-\frac{i\left(s^{4}-1\right)}{s^{2}-1}}\left(s^{4}\right)^{\frac{i\left(s^{6}-1\right)}{s^{2}-1}}\right)$, $0 \leq i \leq s^{4}-s^{2}+1 ;$

5) $L_{s^{14}}\left(\left(s^{7}\right)^{\frac{s^{14}-1}{s^{7}-1}-\frac{i\left(s^{2}-1\right)}{s-1}}\left(s^{2}\right)^{\frac{i\left(s^{7}-1\right)}{s-1}}\right)$,

$$
0 \leq i \leq\left(s^{7}+1\right) /(s+1) \text {; and }
$$

6) $L_{s^{15}}\left(\left(s^{5}\right)^{\frac{s^{15}-1}{s^{5}-1}-\frac{i\left(s^{3}-1\right)}{s-1}}\left(s^{3}\right)^{\frac{i\left(s^{5}-1\right)}{s-1}}\right)$,

$$
0 \leq i \leq\left(s^{10}+s^{5}+1\right) /\left(s^{2}+s+1\right) .
$$

The following theorem gives a set of $s-1$ disjoint $(n-1)$-flats over $G F(s)$ in $P G\left(1, s^{n}\right)$.

Theorem 5. For $i=0, \cdots, s-2$, let $T_{i}=\left\{\left(\gamma, \omega^{i} \gamma^{s}\right): \gamma \in G F\left(s^{n}\right) \backslash\{0\}\right\}$, where $\omega$ is a primitive element of $G F\left(s^{n}\right)$. Then $T_{0}, \cdots, T_{s-2}$, are $s-1$ disjoint $(n-1)$-flats over $G F(s)$ in $P G\left(1, s^{n}\right)$.

Proof. $T_{i}$ is a set of $\left(s^{n}-1\right) /(s-1)$ points in $P G(1$, $\left.s^{n}\right)$, since $\left(\alpha \gamma, \omega^{i}(\alpha \gamma)^{s}\right)\left(=\alpha\left(\gamma, \omega^{i} \gamma^{s}\right)\right)$ represents the same point for each nonzero element $\alpha$ of $G F(s)$. To show that $T_{i}$ is an $(n-1)$-flat over $G F(s)$, we prove that any linear combination of elements in $T_{i}$ is again in $T_{i}$. If $\left(\gamma_{1}, \omega^{i} \gamma_{1}^{s}\right),\left(\gamma_{2}, \omega^{i} \gamma_{2}^{s}\right) \in T_{i}$ and $\alpha_{1}, \alpha_{2} \in G F\left(s^{n}\right)$, then

$$
\begin{aligned}
& \alpha_{1}\left(\gamma_{1}, \omega^{i} \gamma_{1}^{s}\right)+\alpha_{2}\left(\gamma_{2}, \omega^{i} \gamma_{2}^{s}\right) \\
= & \left(\alpha_{1} \gamma_{1}+\alpha_{2} \gamma_{2}, \omega^{i}\left(\alpha_{1} \gamma_{1}+\alpha_{2} \gamma_{2}\right)^{s}\right) \in T_{i} .
\end{aligned}
$$

For $0 \leq i<j \leq s-2$, if $\left(\gamma_{1}, \omega^{i} \gamma_{1}^{s}\right) \in T_{i}$ and $\left(\gamma_{2}, \omega^{j} \gamma_{2}^{s}\right) \in T_{j}$ represent the same points in $P G\left(1, s^{n}\right)$, then $\omega^{i} \gamma_{1}^{s-1}=\omega^{j} \gamma_{2}^{s-1}$. Hence $\omega^{j-i}=\left(\gamma_{1} / \gamma_{2}\right)^{s-1}$. But $\left(\gamma_{1} / \gamma_{2}\right)^{s-1}=\omega^{k(s-1)}$ for some $0 \leq k \leq\left(s^{n}-1\right) /(s-1)-1$, which contradicts $0 \leq i<j \leq s-2$. Hence $T_{i}$ and $T_{j}$ are disjoint for all $0 \leq i<j \leq s-2$.

Corollary 2. $L_{s^{2 n}}\left(\left(s^{n}\right)^{s^{n}+1-i\left(s^{n-1}-1\right) /(s-1)}\left(s^{n-1}\right)^{i\left(s^{n}-1\right) /(s-1)}\right)$ can be constructed for any integer $n$, prime power $s$, and $i=1, \cdots, s-1$.

Proof. We can construct an $L_{s^{2 n}}\left(\left(s^{n}\right)^{s^{n}+1}\right)$ from $P G(1$, $\left.s^{n}\right)$. For each $i=0, \cdots, s-2$, let $T_{i}^{\prime} \subset T_{i}$ be an $(n-2)$ flat over $G F(s)$ in $P G\left(1, s^{n}\right) . \quad\left\{T_{i}^{\prime}: i=0, \cdots, s-2\right\}$ is a set of $s-1$ disjoint ( $n$-2)-flats over $G F(s)$ in $P G\left(1, s^{n}\right)$. Then for each $T_{i}^{\prime}$ we replace the corresponding 
$\left[\left(s^{n-1}-1\right) /(s-1)\right] s^{n}$-level columns in $L_{s^{2 n}}\left(\left(s^{n}\right)^{s^{n}+1}\right)$

by $\left[\left(s^{n}-1\right) /(s-1)\right] s^{n-1}$-level columns to obtain the orthogonal array. $\square$

Example 5. Let $\omega$ be the primitive element of $G F(16)$ with $\omega^{4}=\omega+1$.

$$
\begin{aligned}
T_{0}=\left\{\left(\gamma, \gamma^{2}\right): \gamma \in G F(16) \backslash\{0\}\right\}= & \left\{(1,1),\left(\omega, \omega^{2}\right),\left(\omega^{2}, \omega^{4}\right),\left(\omega^{3}, \omega^{6}\right),\left(\omega^{4}, \omega^{8}\right),\left(\omega^{5}, \omega^{10}\right),\left(\omega^{6}, \omega^{12}\right),\left(\omega^{7}, \omega^{14}\right),\right. \\
& \left.\left(\omega^{8}, \omega\right),\left(\omega^{9}, \omega^{3}\right),\left(\omega^{10}, \omega^{5}\right),\left(\omega^{11}, \omega^{7}\right),\left(\omega^{12}, \omega^{9}\right),\left(\omega^{13}, \omega^{11}\right),\left(\omega^{14}, \omega^{13}\right)\right\}
\end{aligned}
$$

is a 3-flat over $G F(2)$ in $P G(1,16)$ and

$$
T_{0}^{\prime}=\left\{(1,1),\left(\omega, \omega^{2}\right),\left(\omega^{2}, \omega^{4}\right),\left(\omega^{4}, \omega^{8}\right),\left(\omega^{5}, \omega^{10}\right),\left(\omega^{8}, \omega\right),\left(\omega^{10}, \omega^{5}\right)\right\} \subset T_{0}
$$

is a 2-flat over $G F(2)$ in $P G(1,16)$.

Note that we are able to find two disjoint 2-flats over $G F(2)$ in $P G(1,16)$ in Example 2 by trial and error. However, we do not have a method to find more than $s-1$ disjoint (n-2)-flats over $G F(s)$ in $P G\left(1, s^{n}\right)$. With $n=4,5$, 6 and 7 in Corollary 2, we obtain the following new series of tight orthogonal arrays for any prime power $s$ and $i=1, \cdots, s-1$.

1) $L_{s^{8}}\left(\left(s^{4}\right)^{s^{4}+1-i\left(s^{3}-1\right) /(s-1)}\left(s^{3}\right)^{i\left(s^{4}-1\right)}\right)$;

Theorem 6. For any integer $n \geq 2$ and $\beta \in G F\left(s^{n}\right) \backslash\{0\}$,

$$
T_{\beta}=\left\{\left(\gamma, \gamma^{s}, \alpha \beta\right): \gamma \in G F\left(s^{n}\right), \alpha \in G F(s),(\alpha, \gamma) \neq(0,0)\right\}
$$

is an $n$-flat over $G F(s)$ in $P G\left(2, s^{n}\right)$.

However, for $\beta_{1} \neq \beta_{2}$ the $n$-flats over $G F(s) T_{\beta_{1}}$ and $T_{\beta_{2}}$ are not disjoint in $P G\left(2, s^{n}\right)$. But if $s=2$, we can

find more disjoint $n$-flats over $G F(2)$ in $P G\left(2,2^{n}\right)$.

Theorem 7. Let $\omega$ be a primitive element of $G F\left(2^{n}\right)$, and let

$$
\begin{aligned}
& S=\left\{\left(\gamma, \alpha \omega, \gamma^{2}\right): \gamma \in G F\left(2^{n}\right), \alpha \in G F(2),(\alpha, \gamma) \neq(0,0)\right\}, \\
& T=\left\{\left(\gamma, \gamma^{2}, \alpha\right): \gamma \in G F\left(2^{n}\right), \alpha \in G F(2),(\alpha, \gamma) \neq(0,0)\right\}, \\
& U=\left\{\left(\gamma^{2}, \alpha \omega, \gamma\right): \gamma \in G F\left(2^{n}\right), \alpha \in G F(2),(\alpha, \gamma) \neq(0,0)\right\}, \text { and } \\
& V=\left\{\left(\alpha \omega^{2}, \gamma^{2}, \gamma\right): \gamma \in G F\left(2^{n}\right), \alpha \in G F(2),(\alpha, \gamma) \neq(0,0)\right\} .
\end{aligned}
$$

\section{Then we have}

1) $S$ and $T$ are disjoint $n$-flats over $G F(2)$ in $P G\left(2,2^{n}\right)$ for $n \geq 2$.

2) $T, U$ and $V$ are three disjoint n-flats over $G F(2)$ in $P G\left(2,2^{n}\right)$ if $n$ is even.

Proof. By Theorem 6, $S, T, U$ and $V$ are $n$-flats over $G F(2)$ in $P G\left(2,2^{n}\right)$. We now prove that $S$ and $T$ are disjoint. Assume that $\left(\gamma_{1}, \alpha_{1} \omega, \gamma_{1}^{2}\right) \in S$ and $\left(\gamma_{2}, \gamma_{2}^{2}, \alpha_{2}\right) \in T$ represent the same point in $P G\left(2,2^{n}\right)$, where $\alpha_{1}, \alpha_{2} \in G F(2)$ and $\gamma_{1}, \gamma_{2} \in G F\left(2^{n}\right)$. Clearly, $\alpha_{1}, \alpha_{2}, \gamma_{1}, \gamma_{2} \neq 0$, hence $\alpha_{1}=\alpha_{2}=1$ and $\left(\gamma_{1}, \omega, \gamma_{1}^{2}\right)$ and $\left(\gamma_{2}, \gamma_{2}^{2}, 1\right)$ represent the same point. We have $\omega / \gamma_{1}=\gamma_{2}$ and $\gamma_{1}=1 / \gamma_{2}$, which imply $\omega=1$, a contradiction. Hence $S$ and $T$ are disjoint. Now we show that $T$ and $U$ are disjoint if $n$ is even. If $n$ is even then 3 divides $2^{n}-1$. For any $\gamma \in G F\left(2^{n}\right) \backslash\{0\}, \gamma^{3}=\omega^{3 k}$ for some $0 \leq k \leq\left(2^{n}-1\right) / 3-1$. Assume that $\left(\gamma_{2}, \gamma_{2}^{2}, \alpha_{2}\right) \in T$ and $\left(\gamma_{3}^{2}, \alpha_{3} \omega, \gamma_{3}\right) \in U$ represent the same point in $P G\left(2,2^{n}\right)$, where $\alpha_{2}, \alpha_{3} \in G F(2)$ and $\gamma_{2}, \gamma_{3} \in G F\left(2^{n}\right)$. Clearly, $\alpha_{2}$, $\alpha_{3}, \gamma_{2}, \gamma_{3} \neq 0$, hence $\alpha_{2}=\alpha_{3}=1$ and $\left(\gamma_{2}, \gamma_{2}^{2}, 1\right)$ and

$\left(\gamma_{3}^{2}, \omega, \gamma_{3}\right)$ represent the same point. We have $\gamma_{2}=\gamma_{3}$ and $\gamma_{2}^{2}=\omega / \gamma_{3}$, which imply $\omega=\gamma_{2}^{3}=\omega^{3 k}$ for some $0 \leq k \leq\left(2^{n}-1\right) / 3-1$, a contradiction. Hence $T$ and $U$ are disjoint. We can similarly prove that $T$ and $V$ are disjoint and that $U$ and $V$ are disjoint if $n$ is even. $\square$

An $L_{s^{3 n}}\left(\left(s^{n}\right)^{s^{2 n}+s^{n}+1}\right)$ can be constructed from $P G(2$, $s^{n}$ ). By applying Theorems 2, 6, and 7, we obtain the fol- 
lowing orthogonal arrays.

Corollary 3. For any prime power $s$, we can construct

1) $L_{s^{3 n}}\left(\left(s^{n+1}\right)^{\left(s^{n}-1\right) /(s-1)}\left(s^{n}\right)^{s^{2 n}-\left(s^{n}-s\right) /(s-1)}\right), n \geq 2$;
2) $L_{2^{3 n}}\left(\left(2^{n+1}\right)^{2^{n+1}-2}\left(2^{n}\right)^{2^{2 n}-3 \cdot 2^{n}+3}\right), n \geq 2$; and

3) $L_{2^{6 n}}\left(\left(2^{2 n+1}\right)^{3 \cdot 2^{2 n}-3}\left(2^{2 n}\right)^{2^{4 n}-5 \cdot 2^{n}+4}\right), n \geq 1$

Example 6. Let $\omega$ be the primitive element of $G F(8)$ with $\omega^{3}=\omega+1$. Let

$$
\begin{aligned}
S=\{ & (1,1,0),\left(\omega, \omega^{2}, 0\right),\left(\omega^{2}, \omega^{4}, 0\right),\left(\omega^{3}, \omega^{6}, 0\right),\left(\omega^{4}, \omega, 0\right),\left(\omega^{5}, \omega^{3}, 0\right),\left(\omega^{6}, \omega^{5}, 0\right),(0,0,1), \\
& \left.(1,1,1),\left(\omega, \omega^{2}, 1\right),\left(\omega^{2}, \omega^{4}, 1\right),\left(\omega^{3}, \omega^{6}, 1\right),\left(\omega^{4}, \omega, 1\right),\left(\omega^{5}, \omega^{3}, 1\right),\left(\omega^{6}, \omega^{5}, 1\right)\right\} \\
T=\{ & (1,0,1),\left(\omega, 0, \omega^{2}\right),\left(\omega^{2}, 0, \omega^{4}\right),\left(\omega^{3}, 0, \omega^{6}\right),\left(\omega^{4}, 0, \omega\right),\left(\omega^{5}, 0, \omega^{3}\right),\left(\omega^{6}, 0, \omega^{5}\right),(0, \omega, 0), \\
& \left.(1, \omega, 1),\left(\omega, \omega, \omega^{2}\right),\left(\omega^{2}, \omega, \omega^{4}\right),\left(\omega^{3}, \omega, \omega^{6}\right),\left(\omega^{4}, \omega, \omega\right),\left(\omega^{5}, \omega, \omega^{3}\right),\left(\omega^{6}, \omega, \omega^{5}\right)\right\}
\end{aligned}
$$

be two disjoint 3-flats over $G F(2)$ in $P G(2,8)$. An $L_{512}\left(8^{73}\right)$ can be constructed from $P G(2,8)$. We can replace the subarray $L_{512}\left(8^{15}\right)$ corresponding to $S$ or $T$ by an $L_{512}\left(16^{7}\right)$ to obtain $L_{512}\left(16^{7} 8^{58}\right)$ and $L_{512}\left(16^{14} 8^{43}\right)$. $\square$

The following two examples are obtained by applying Theorems 3 and 5 and by trial and error.

Example 7. Let $\omega$ be the primitive element of $G F(8)$ with $\omega^{3}=\omega+1$. Let

$$
\begin{aligned}
& A_{1}=\left\{\left(1, \omega, \omega^{2}\right),\left(0,1, \omega^{2}\right),\left(1, \omega^{3}, 0\right)\right\}, \\
& B_{1}=\left\{\left(1, \omega^{2}, \omega^{4}\right),\left(0,1, \omega^{4}\right),\left(1, \omega^{6}, 0\right)\right\}, \\
& C_{1}=\left\{\left(1, \omega^{4}, \omega\right),(0,1, \omega),\left(1, \omega^{5}, 0\right)\right\},
\end{aligned}
$$

and $W$ be the $3 \times 3$ matrix defined in Example 1. For $i=2, \cdots, 7$, let $A_{i}$ (or $B_{i}, C_{i}$ ) be the set obtained by multiplying each element in $A_{i}$ (or $B_{i}, C_{i}$ ) by $W$. For Example,

$$
A_{2}=\left\{\left(1, \omega, \omega^{2}\right) W,\left(0,1, \omega^{2}\right) W,\left(1, \omega^{3}, 0\right) W\right\}=\left\{\left(\omega^{2}, \omega^{6}, \omega\right),\left(\omega^{2}, \omega^{2}, 1\right),\left(0,1, \omega^{3}\right)\right\} .
$$

It can be verified that $A_{1}, \cdots, A_{7}, B_{1}, \cdots, B_{7}, C_{1}, \cdots, C_{7}$, and $\{(1,0,0),(0,1,0),(1,1,0)\}$ are 22 disjoint 1-flats over $G F(2)$ in $P G(2,8)$. An $L_{512}\left(8^{73}\right)$ can be constructed from $P G(2,8)$. We can replace the subarray $L_{512}\left(8^{3}\right)$ corresponding to each 1-flat over $G F(2)$ in $P G(2,8)$ by an $L_{512}\left(4^{7}\right)$ to obtain $L_{512}\left(8^{73-3 i} 4^{7 i}\right)$ for $i=1, \cdots, 22$. $\square$

Example 8. Let $\omega$ be the primitive element of $G F(32)$ with $\omega^{5}=\omega^{2}+1$. An $L_{1024}\left(32^{33}\right)$ can be constructed from $P G(1,32)$.

1)

$$
\begin{aligned}
& A_{1}=\{(1,0),(0,1),(1,1)\}, \\
& A_{2}=\left\{(1, \omega),\left(\omega, \omega^{18}\right),\left(\omega^{18}, 1\right)\right\}, \\
& A_{3}=\left\{\left(1, \omega^{2}\right),\left(\omega^{2}, \omega^{5}\right),\left(\omega^{5}, 1\right)\right\}, \\
& A_{4}=\left\{\left(1, \omega^{4}\right),\left(\omega^{4}, \omega^{10}\right),\left(\omega^{10}, 1\right)\right\},
\end{aligned}
$$

$$
\begin{aligned}
& A_{5}=\left\{\left(1, \omega^{5}\right),\left(\omega^{5}, \omega^{2}\right),\left(\omega^{2}, 1\right)\right\}, \\
& A_{6}=\left\{\left(1, \omega^{8}\right),\left(\omega^{8}, \omega^{20}\right),\left(\omega^{20}, 1\right)\right\}, \\
& A_{7}=\left\{\left(1, \omega^{9}\right),\left(\omega^{9}, \omega^{16}\right),\left(\omega^{16}, 1\right)\right\}, \\
& A_{8}=\left\{\left(1, \omega^{10}\right),\left(\omega^{10}, \omega^{4}\right),\left(\omega^{4}, 1\right)\right\}, \\
& A_{9}=\left\{\left(1, \omega^{14}\right),\left(\omega^{14}, \omega^{13}\right),\left(\omega^{13}, 1\right)\right\}, \\
& A_{10}=\left\{\left(1, \omega^{16}\right),\left(\omega^{16}, \omega^{9}\right),\left(\omega^{9}, 1\right)\right\}, \text { and } \\
& A_{11}=\left\{\left(1, \omega^{19}\right),\left(\omega^{19}, \omega^{11}\right),\left(\omega^{11}, 1\right)\right\}
\end{aligned}
$$

are eleven disjoint 1-flats over $G F(2)$ in $P G(1,32)$. We can replace the subarray $L_{1024}\left(32^{3}\right)$ corresponding to each 1-flat over $G F(2)$ in the $L_{1024}\left(32^{33}\right)$ by an $L_{1024}\left(16^{3} 4^{16}\right)$ to obtain $L_{1024}\left(32^{33-3 i} 16^{3 i} 4^{16 i}\right)$ for $i=1, \cdots, 11$.

2)

$$
\begin{aligned}
& B_{1}=\left\{\left(1, \omega^{21}\right),\left(\omega, \omega^{23}\right),\left(\omega^{18}, \omega^{26}\right),\left(\omega^{2}, \omega^{25}\right),\left(\omega^{5}, 1\right),\left(\omega^{19}, \omega^{28}\right),\left(\omega^{11}, \omega^{12}\right)\right\}, \\
& B_{2}=\left\{\left(1, \omega^{25}\right),\left(\omega, \omega^{8}\right),\left(\omega^{18}, \omega^{7}\right),\left(\omega^{2}, \omega^{14}\right),\left(\omega^{5}, \omega^{2}\right),\left(\omega^{19}, \omega^{4}\right),\left(\omega^{11}, \omega^{29}\right)\right\}, \\
& B_{3}=\left\{\left(1, \omega^{2}\right),\left(\omega, \omega^{5}\right),\left(\omega^{18}, 1\right),\left(\omega^{2}, \omega^{16}\right),\left(\omega^{5}, \omega^{15}\right),\left(\omega^{19}, \omega^{24}\right),\left(\omega^{11}, \omega^{9}\right)\right\}, \text { and } \\
& B_{4}=\left\{\left(1, \omega^{6}\right),\left(\omega, \omega^{25}\right),\left(\omega^{18}, \omega^{17}\right),\left(\omega^{2}, \omega^{19}\right),\left(\omega^{5}, \omega^{20}\right),\left(\omega^{19}, \omega^{15}\right),\left(\omega^{11}, \omega^{22}\right)\right\}
\end{aligned}
$$

are four disjoint 2-flats over $G F(2)$ in $P G(1,32)$. We can replace the subarray $L_{1024}\left(32^{7}\right)$ corresponding to each 2- flat over $G F(2)$ in the $L_{1024}\left(32^{33}\right)$ by an $L_{1024}\left(16^{7} 8^{16}\right)$ or an $L_{1024}\left(8^{31}\right)$ to obtain $L_{1024}\left(32^{33-7 i-7 j} 16^{7 i} 8^{16 i+31 j}\right)$ for $1 \leq i+j \leq 4$. 
3)

$$
\begin{aligned}
& C_{1}=B_{1} \cup\left\{\left(\omega^{3}, \omega^{27}\right),\left(\omega^{29}, \omega^{17}\right),\left(\omega^{6}, \omega^{2}\right),\left(\omega^{27}, \omega^{13}\right),\left(\omega^{20}, \omega^{30}\right),\left(\omega^{8}, \omega^{6}\right),\left(\omega^{12}, \omega^{14}\right),\left(\omega^{23}, \omega^{5}\right)\right\} \text { and } \\
& C_{2}=B_{2} \cup\left\{\left(\omega^{3}, \omega^{18}\right),\left(\omega^{29}, \omega^{9}\right),\left(\omega^{6}, \omega^{12}\right),\left(\omega^{27}, \omega^{26}\right),\left(\omega^{20}, \omega^{24}\right),\left(\omega^{8}, \omega^{11}\right),\left(\omega^{12}, \omega^{17}\right),\left(\omega^{23}, \omega^{6}\right)\right\}
\end{aligned}
$$

are two disjoint 3-flats over $G F(2)$ in $P G(1,32)$, where $B_{1}$ and $B_{2}$ are 2-flats over $G F(2)$ in 2). Moreover, $C_{1}, C_{2}$, and $A_{1}$ in 1) partition $P G(1,32)$. We can replace the subarray $L_{1024}\left(32^{15}\right)$ corresponding to $C_{1}$ or $C_{2}$ in the $L_{1024}\left(32^{33}\right)$ by an $L_{1024}\left(16^{24} 8^{15}\right)$ or an $L_{1024}\left(16^{31}\right)$ to obtain $L_{1024}\left(32^{18} 16^{24} 8^{15}\right), L_{1024}\left(32^{3} 16^{48} 8^{30}\right), L_{1024}\left(32^{18} 16^{31}\right)$, $L_{1024}\left(32^{3} 16^{62}\right)$, and $L_{1024}\left(32^{3} 16^{55} 8^{15}\right)$.

\section{Discussion}

We use $t$-flats over $G F(s)$ in $P G\left(m-1, s^{n}\right)$ to find different ways to regroup a set of $(n-1)$-flats in $P G\left(m-1, s^{n}\right)$ into disjoint flats. However, many problems remain unsolved. For example, we do not know how many disjoint $(n-2)$-flats over $G F(s)$ exist in $P G\left(1, s^{n}\right)$. Since there are $s^{n}+1$

$\left(=\left(s^{2}-s\right)\left(s^{n-1}-1\right) /(s-1)+s+1\right)$ points in $P G\left(1, s^{n}\right)$, the upper bound for the number of disjoint $(n-2)$-flats over $G F(s)$ equals $s^{2}-s$ if $n \geq 4$ and equals $s^{2}-s+1$ if $n=$ 3 . An obvious conjecture is that $P G\left(1, s^{n}\right)$ can be partitioned into $\left(s^{2}-s\right)(n-2)$-flats and one 1-flat over $G F(s)$. This conjecture is true for $n=3$, since $P G\left(1, s^{3}\right)$ can be partitioned into $\left(s^{2}-s+1\right)$ 1-flats over $G F(s)$ by Theorem 4. It is also true for $s=2$ and $n=4,5$, which are shown in Example 2 for $n=4$ and shown in Example 8(3) for $n=5$. If the conjecture is true, we can construct an $L_{s^{2 n}}\left(\left(s^{n}\right)^{s^{n}+1-i\left(s^{n-1}-1\right) /(s-1)}\left(s^{n-1}\right)^{i\left(s^{n}-1\right) /(s-1)}\right)$ for $n \geq 3$ and $i=1, \cdots, s^{2}-s$, which would be a significant improvement of Corollary 2.

Also we do not know how many disjoint $n$-flats over $G F(s)$ exist in $P G\left(2, s^{n}\right)$. The number of points in $P G(2$, $\left.s^{n}\right)$ is

$$
s^{2 n}+s^{n}+1=\left(s^{n}-s^{n-1}\right)\left(s^{n+1}-1\right) /(s-1)+s^{n}+s^{n-1}+1 .
$$

Hence an upper bound for the number of disjoint $n$-flats over $G F(s)$ in $P G\left(2, s^{n}\right)$ is $s^{n}-s^{n-1}$ if $n \geq 3$ and is $s^{2}-s+$ 1 if $n=2$. The upper bound is attained for $n=2$, since $P G\left(2, s^{n}\right)$ can be partitioned into $\left(s^{2}-s+1\right)$ 2-flats over $G F(s)$ by Theorem 4. In general, the difference between the upper bound and what can be obtained in Theorems 6 and 7 is considerably large for $n \geq 3$. There may be better ways to find disjoint $n$-flats over $G F(s)$ in $P G\left(2, s^{n}\right)$ than the approach used in Theorems 6 and 7. So far, we do not know any example having $s^{n}-s^{n-1}$ disjoint $n$-flats over $G F(s)$ in $P G\left(2, s^{n}\right)$ for $n \geq 3$.

Another problem which cannot be solved by the ap- proach of this paper is the construction of orthogonal arrays having $s^{n}$ rows, where $n$ is a prime number. For example, it is known that $L_{128}\left(16^{1} 8^{16}\right)$ can be constructed by a mixed spread of $P G(6,2)$, which consists of a 3 -flat and 16 2-flats. But it is not known that if it is possible to find, among those 16 2-flats, disjoint sets of three 2-flats such that each set can be regrouped into seven 1-flats. We could construct an $L_{128}\left(16^{1} 8^{16-3 i} 4^{7 i}\right)$ if there exist $i$ such disjoint sets of three 2-flats.

\section{REFERENCES}

[1] R. C. Bose and K. A. Bush, "Orthogonal Arrays of Strength Two and Three," The Annals of Mathematical Statistics, Vol. 23, No. 4, 1952, pp. 508-524. doi:10.1214/aoms/1177729331

[2] R. L. Plackett and J. P. Burma, "The Design of Optimum Multifactorial Experiments," Biometrika, Vol. 33, No. 4, 1946, pp. 305-325.

[3] C. R. Rao, "Factorial Experiments Derivable from Combinatorial Arrangements of Arrays," Journal of Royal Statistical Society (Supplement), Vol. 9, No. 1, 1947, pp. 128-139. doi: $10.2307 / 2983576$

[4] C. R. Rao, "Some Combinatorial Problems of Arrays and Applications to Design of Experiments," In: J. N. Srivastava, Ed., A Survey of Combinatorial Theory, NorthHolland, Amsterdam, 1973, pp. 349-359.

[5] J. C. Wang and C. F. J. Wu, "An Approach to the Construction of Asymmetrical Orthogonal Arrays," Journal of American Statistical Association, Vol. 86, No. 414, 1991, pp. 450-456. doi:10.2307/2290593

[6] C. F. J. Wu, R. C. Zhang and R. Wang, "Construction of Asymmetrical Orthogonal Array of Type $O A\left(s^{k}, s^{m}\left(s^{r 1}\right)^{n 1} \ldots\right.$ $\left.\left(s^{r t}\right)^{n t}\right)$, , Statistica Sinica, Vol. 2, No. 1, 1992, pp. 203219.

[7] A. Dey and C. K. Midha, "Construction of Some Asymmetrical Orthogonal Arrays," Statistics \& Probability Letters, Vol. 28, No. 3, 1996, pp. 211-217. doi:10.1016/0167-7152(95)00126-3

[8] Y. S. Zhang, Y. Q. Lu and S. Q. Pang, "Orthogonal Arrays Obtained by Orthogonal Decomposition of Projection Matrices," Statistica Sinica, Vol. 9, No. 2, 1999, pp. 595-604.

[9] C. Suen and W. F. Kuhfeld, "On the Construction of Mixed Orthogonal Arrays of Strength Two," Journal of Statistical Planning and Inference, Vol. 133, No. 2, 2005, pp. 555-560. doi:10.1016/j.jspi.2004.03.018

[10] A. S. Hedayat, N. J. A. Sloane and J. Stufken, "Orthogonal Arrays," Springer, New York, 1999. doi:10.1007/978-1-4612-1478-6

[11] S. Addelman, "Orthogonal Main Effect Plans for Asym- 
metrical Factorial Experiments," Technometrics, Vol. 4, No. 1, 1962, pp. 21-46. doi:10.2307/1266170

[12] C. F. J. Wu, "Construction of $2^{m} 4^{n}$ Deigns via a Grouping Scheme," Annals of Statistics, Vol. 17, No. 4, 1989, pp. 1880-1885. doi:10.1214/aos/1176347399

[13] E. M. Rains, N. J. A. Sloane and J. Stufken, "The Lattice of $N$-Run Orthogonal Arrays," Journal of Statistical Planning and Inference, Vol. 102, No. 2, 2002, pp. 477-500. doi:10.1016/S0378-3758(01)00119-7
[14] C. Suen, A. Das and A. Dey, "On the Construction of Asymmetric Orthogonal Arrays," Statistica Sinica, Vol. 11, No. 1, 2001, pp. 241-260.

[15] J. W. P. Hirschefld, "Projective Geometries over Finite Fields," Oxford University Press, Oxford, 1979.

[16] C. Suen and A. Dey, "Construction of Asymmetric Orthogonal Arrays through Finite Geometries," Journal of Statistical Planning and Inference, Vol. 115, No. 2, 2003, pp. 623-635. doi:10.1016/S0378-3758(02)00165-9 